monitoring and commissioned 'BritainThinks' to conduct four focus groups and ten in-depth interviews with the public, dying people and carers.

Results A number of key findings emerged from a thematic analysis of the research:

- people are not always given clear information about their symptoms, prognosis or treatment options

- when people want more information they do not always know the right questions to ask in order to obtain it

- people are not always supported to make meaningful choices at the end of life

- the extent to which people are empowered to make informed decisions about their care can have a direct impact on care quality

- opportunities to instigate conversations about future care are being missed.

Conclusions We are using the extensive data we have obtained to:

- update our policy calls

- develop resources to give people tools to improve their own care

- enhance our service development, including adding more clinical expertise to our information line.

Our study demonstrates the tangible thread that links learning from dying people and improving service delivery. We encourage others in the sector to undertake similar work.

\section{P-90 IMPLEMENTATION OF RESEARCH INTO HOSPICES THROUGH COMMUNITY OF INFLUENCE: CRN WEST MIDLANDS MODEL}

Sandra Prew, Marion Evans, Rebecca Burt, Julie Machin, Clare Marlow, Andrea Shilton, Ami Salter, Annie Young, Marie Phipps, Hilary Shepley. Clinical Research Network, West Midlands, UK

\subsection{6/bmjspcare-2017-hospice.117}

There is a need to provide high quality, evidence-based supportive and palliative care research. However, recruitment to supportive and palliative care research studies remains a challenge. Therefore, more research evidence is required in this specialty. The supportive and palliative care research model aims to:

- Improve care through research, increase awareness of research within the West Midlands and share nationally

- Support local hospices and care homes to become involved in research

- Encourage clinicians to identify topics for future research, to be adopted onto the National Research Portfolio, and ensure that researchers in the area are able to offer access to studies for participants in all areas.

- Progress and achievements to date include:

- contributing to the delivery of existing National Institute for Health Research Portfolio studies by carrying out site initiation visits and assisting with study recruitment.

- generating new studies that are running in the West Midlands (WM) through the WM Collaborative Actioning Research in End of Life and Supportive Care (WMCARES). sharing research priority topics identified following a survey of hospices in the West Midlands and to investigate in the near future.
- increasing our visibility across the West Midlands to promote and share our strategy. This has involved talks with local radio, West Midlands partner organisations, other Clinical Research Networks and primary care organisations.

- bringing together an enthusiastic and committed workforce of diverse healthcare professionals, including clinical research speciality and sub-specialty leads, a nursing team, locality manager, administrative staff, research delivery and portfolio managers.

Our next planned steps include short and long term goals. Highlights include: Enhanced IT systems, develop a Health Research Authority flow diagram and create a Supportive and Palliative Care Nurse Forum with collaboration with CRN West Midlands.

\section{Patient care}

\section{P-91 DO COMPLEMENTARY THERAPIES WORK?}

Sally Baker, Candy Kitz-Atsma, Russ Hargreaves. ellenor, Gravesend, UK

\subsection{6/bmjspcare-2017-hospice.118}

Background Complementary therapies are becoming routinely offered in hospices and other healthcare settings throughout the UK with as many as $51 \%$ of patients accessing therapies (Posadzki et al., 2013). However, little objective data about effectiveness exists making them difficult to justify in an evidence-based healthcare system. This study used the validated MYCAW tool (Measure Yourself Concerns and Wellbeing) in an attempt to explore the value of massage, aromatherapy and reflexology in a range of patients and carers (Jolliffe et al., 2014; Polley, 2007; Seers, 2009).

Aims The aim of the project was two-fold:

1. To assess the practicalities of implementing MYCAW in a hospice-setting

2. To assess the use of MYCAW following an intervention to improve its use.

Methods Over a one-year period, 98 patients and carers were assessed using MYCAW pre- and post-complementary therapies.

Results Complete data was only available for 32\% of subjects. Incomplete data resulted from failure to record unique identifier on the MYCAW form (6\%), clients failing to attend all of their scheduled therapies (55\%) and patient death resulting in a failure to record a second set of MYCAW data (7\%). Because the data were limited, a new protocol was implemented in the hope of improving the use of MYCAW. An electronic diary system was introduced with prompts for all first and last MYCAW. Meetings were held with all therapists to reinforce the importance of MYCAW. We also created 'appointment packs' containing MYCAW forms to further prompt therapists. As a result of this intervention, we have seen a dramatic improvement in the number of completed MYCAW forms both pre- and post-therapy. Early data suggest that MYCAW is a useful tool to assess client-defined worries. Conclusion The data suggest complementary therapies are an effective way of reducing patients' and carers' concerns in a hospice-setting. 